\title{
Nominata de Avaliadores ad hoc 2011
}

1. Adailton Maciel Augusto - Instituto São Paulo de Estudos Superiores

2. Adilson José Francisco - UFMT

3. Adriana Sampaio Evangelista - UFJF

4. Agemir de Carvalho Dias - FEPAR

5. Agnaldo Cuoco Portugal - UnB

6. Airton Luiz Jungblut - PUC-RS

7. Alexandra Santos Pinheiro - UFGD

8. Alexandre Brasil Carvalho da Fonseca - UFRJ

9. Alexandre Mansur - UFJF

10. Alfredo dos Santos Oliva - UEL

11. Antonia Aparecida Quintão dos Santos Cezerilo - Mackenzie-SP

12. Antônio Geraldo Cantarela - PUC-Minas

13. Antônio Mendes da Costa Braga - Unicamp

14. Antonio Paulo Benatte - UEPG

15. Antônio Thadeu de Oliveira Xavier - Universidade Católica do Salvador

16. Aparecida de Fátima Bueno - USP

17. Beliza Áurea de Arruda Mello - UFPB

18. Benedito Ferraro - PUC Campinas

19. Berta Lúcia Pinheiro Klüppel - UFPB

20. Brenda Maribel Carranza Dávila - PUC Campinas

21. Carlos João Tavares Nunes Correia - Universidade de Lisboa (Portugal) 
22. Claudio de Oliveira Ribeiro - UMESP

23. Daniel Alves - UFG

24. Drance Elias da Silva - UNICAP

25. Eliane Martins de Oliveira - Fund. de Apoio à Esc.Téc. do Est. Rio de Janeiro

26. Elias Wolff - Instituto Teológico de Santa Catarina - ITESC

27. Ênio Brito Pinto - PUC-SP

28. Edebrande Cavalieri - UFES

29. Eufrázia Cristina Menezes Santos - UFS

30. Everaldo Cescon - Universidade de Caxias do Sul

31. Faustino Luiz Couto Teixeira - UFJF

32. Flávio Henrique Dias Saldanha - UFTM

33. Flávio Munhoz Sofiati - UFG

34. Flávio Rodrigo Freire Ferreira - Faculdade Natalense de Ensino e Cultura

35. Francisco de Barros Barbosa - Universidade Católica de Salvador

36. Francisco das Chagas de Albuquerque - FAJE

37. Gilbraz de Souza Aragão - UNICAP

38. Gilmar Rocha - UFF

39. Giovana Calcagno Gomes - FURG

40. Gisela Isolde Waechter Streck - Escola Superior de Teologia - EST

41. Gizele Zanotto - UPF

42. Grayce Mayre Bonfim Souza - UESB

43. Hermisten Maia Pereira da Costa - Mackenzie -Rio

44. Irene de Araújo van den Berg - UERN

45. Jérri Roberto Marin - UFMS

46. João Augusto Anchieta Amazonas Mac Dowell - FAJE 
47. João Batista Libanio - FAJE

48. João Marcus Figueiredo Assis - UNIRIO

49. Joe Marçal Gonçalves dos Santos - PUC-RS

50. Jonatas Silva Meneses - UFS

51. Jorge Claudio Noel Ribeiro Júnior - PUC-SP

52. José Bittencourt Filho - UnB

53. José Carlos Aguiar de Souza - PUC-Minas

54. José Francisco Miguel Henriques Bairrão - USP

55. José Neivaldo de Souza - FEPAR

56. José Rogério Machado de Paula - Centro Universitário Salesiano - Lins/SP

57. Juliana de Mello Moraes - Universidade de Lisboa (Portugal)

58. Júlio César Moreno - Centro Universitário Salesiano de São Paulo

59. Karla Denise Martins - UFV

60. Karla Regina Macena Pereira Patriota - UFPE

61. Lemuel Dourado Guerra Sobrinho - UFCG

62. Leomar Antônio Brustolin - PUC-RS

63. Leonildo Silveira Campos - UMESP

64. Lidice Meyer Pinto Ribeiro - Mackenzie - SP

65. Lindomar Rocha Mota - PUC-Minas

66. Luciana Duccini - UNIVASF

67. Luciene Cristina de Oliveira - UFMA

68. Luis Sérgio Krausz - USP

69. Luiz Alencar Libório - UNICAP

70. Luiz Lima Vailati - UFV

71. Lyndon de Araújo Santos - UFMA 
72. Magali do Nascimento Cunha - UMESP

73. Manuel do Carmo da Silva Campos - Universidade do Estado do Amazonas

74. Mara Regina do Nascimento - UFU

75. Marcelo Ayres Camurça Lima - UFJF

76. Marcelo Tavares Natividade - UFC

77. Marcelo Martins Barreira - UFES

78. Maria Clara Lucchetti Bingemer - PUC-Rio

79. Maria de Fátima Paz Alves - UFRPE

80. Maria Helena Nery Garcez - USP

81. Maria Suely Kofes - Unicamp

82. Maria Tereza Santana da Costa R. Almeida - UFPB

83. Marta Rosa Borin - UFSM

84. Mário Antonio Sanches - PUC-PR

85. Mario Feijo Borges Monteiro - UFRJ

86. Marisete Teresinha Hoffmann Horochovski - UFPR

87. Maristela Oliveira de Andrade - UFPB

88. Mary Rute Gomes Esperandio - PUC-PR

89. Miguel Attie Filho - USP

90. Mísia Lins Reesink - UFPE

91. Névio de Campos - UEPG

92. Newton Darwin de Andrade Cabral - UNICAP

93. Orivaldo Pimentel Lopes Junior - UFRN

94. Paulo Donizéti Siepierski - UFRPE

95. Paulo Gracino - Universidade de Vila Velha

96. Paulo Sérgio Lopes Gonçalves - PUC Campinas 
97. Pedro Ribeiro de Oliveira - PUC-Minas

98. Pericles Morais de Andrade Junior - UFS

99. Raymundo Heraldo Maués - UFMA

100. Renato Kirchner - PUC Campinas

101. Ricardo Bitun - Mackenzie - SP

102. Richard Gonçalves André - Universidade Estadual de Maringá

103. Roberlei Panasiewicz - PUC-Minas

104. Roberta Bivar Carneiro Campos - UFPE

105. Roberta Rotta Messias de Andrade - Faculdade Sumaré

106. Robson Medeiros Alves - PUC-SP

107. Rodrigo Coppe Caldeira - PUC-Minas

108. Rodrigo Portella - UFJF

109. Rosalie Helena de Souza Pereira - PUC-SP

110. Salma Ferraz de Azevedo de Oliveira - UFSC

111. Sandro Guimarães de Salles - UFPE

112. Saul Kirschbaum - USP

113. Scott Randall Paine - UnB

114. Sergio Figueiredo Ferretti - UFMA

115. Sergio Néstor Osório García - Universidad Nueva Granada (Colômbia)

116. Severino Vicente da Silva - UFPE

117. Silas Guerriero - PUC-SP

118. Sílvio José Benelli - UNESP

119. Solange Ramos de Andrade - Universidade Estadual de Maringá

120. Sonia Maria Giacomini - PUC-Rio

121. Sulivan Charles Barros - UnB 
122. Suzana dos Santos Gomes - UFMG

123. Tânia Welter - UFSC

124. Tiago Hideo Barbosa Watanabe - UNESP Assis (SP)

125. Valentina Ayrolo - Universidad Nacional de Mar del Plata (Argentina)

126. Vinicius Mariano de Carvalho - University of Aarhus (Dinamarca)

127. Wagner Lopes Sanchez - PUC-SP

128. Wander de Lara Proença - Faculdade Teológica Sul Americana

129. Wania Amélia Belchior Mesquita - UFRJ

130. Willian de Souza Martins - UFRJ

131. Zuleica Dantas Pereira Campos - UNICAP 